\title{
Disruption of posteromedial large-scale neural communication predicts recovery from coma OPEN
}

Stein Silva, MD, PhD

Francesco de Pasquale,

$\mathrm{PhD}$

Corine Vuillaume, MD

Beatrice Riu, MD

Isabelle Loubinoux, $\mathrm{PhD}$

Thomas Geeraerts, MD,

$\mathrm{PhD}$

Thierry Seguin, MD

Vincent Bounes, MD,

$\mathrm{PhD}$

Olivier Fourcade, MD,

$\mathrm{PhD}$

Jean-Francois Demonet,

$\mathrm{MD}, \mathrm{PhD}$

Patrice Péran, PhD

Correspondence to

Dr. Silva:

silva.s@chu-toulouse.fr

\section{Supplemental data} at Neurology.org

\section{ABSTRACT}

Objective: We hypothesize that the major consciousness deficit observed in coma is due to the breakdown of long-range neuronal communication supported by precuneus and posterior cingulate cortex (PCC), and that prognosis depends on a specific connectivity pattern in these networks.

Methods: We compared 27 prospectively recruited comatose patients who had severe brain injury (Glasgow Coma Scale score <8; 14 traumatic and 13 anoxic cases) with 14 agematched healthy participants. Standardized clinical assessment and fMRI were performed on average $4 \pm 2$ days after withdrawal of sedation. Analysis of resting-state fMRI connectivity involved a hypothesis-driven, region of interest-based strategy. We assessed patient outcome after 3 months using the Coma Recovery Scale-Revised (CRS-R).

Results: Patients who were comatose showed a significant disruption of functional connectivity of brain areas spontaneously synchronized with PCC, globally notwithstanding etiology. The functional connectivity strength between PCC and medial prefrontal cortex (mPFC) was significantly different between comatose patients who went on to recover and those who eventually scored an unfavorable outcome 3 months after brain injury (Kruskal-Wallis test, $p<0.001$; linear regression between CRS-R and PCC-mPFC activity coupling at rest, Spearman $\rho=0.93, p<0.003$ ).

Conclusion: In both etiology groups (traumatic and anoxic), changes in the connectivity of PCC-centered, spontaneously synchronized, large-scale networks account for the loss of external and internal self-centered awareness observed during coma. Sparing of functional connectivity between PCC and mPFC may predict patient outcome, and further studies are needed to substantiate this potential prognosis biomarker. Neurology ${ }^{\circledR} 2015 ; 85: 2036-2044$

\section{GLOSSARY}

BOLD = blood oxygen level-dependent; CRS-R = Coma Recovery Scale-Revised; DMN = default-mode network; FDR = false discovery rate; $\mathbf{m P F C}=$ medial prefrontal cortex; $\mathbf{P C C}=$ posterior cingulate cortex; PMC = posteromedial cortex; PreCu = precuneus; $\mathbf{R O I}=$ region of interest; $\mathbf{S M G}=$ supramarginal gyrus; $\mathbf{T B I}=$ traumatic brain injury .

Posteromedial cortex (PMC) has traditionally received little attention from neurologists, ${ }^{1,2}$ possibly as a consequence of the paucity of focal vascular lesions in this region and its concealed localization. Nevertheless, recent findings suggest that it could have a central role in the regulation of consciousness in humans. First, it has dense structural and functional connections suggesting a role as a cortical hub, ${ }^{3}$ an essential property for complex cognitive processes. ${ }^{4}$ Second, PMC is also one of the most metabolically active brain regions, both at rest and during self-directed cognition. ${ }^{5}$ Third, a selective hypometabolism in this structure has been reported in a wide range of altered consciousness states such as sleep, ${ }^{6}$ drug-induced anesthesia, ${ }^{7}$ or chronic disorders of consciousness. ${ }^{89}$ Crucially, in patients with disorders of consciousness, resting-state

\footnotetext{
From the Critical Care Unit (S.S., B.R.), Critical Care and Anaesthesiology Department (S.S., C.V., B.R., T.G., T.S., O.F.), and SAMU 31 (V.B.), University Teaching Hospital of Purpan, Place du Dr Baylac, F-31059 Toulouse Cedex 9; INSERM U825 (S.S., C.V., I.L., P.P.), CHU Purpan, Place du Dr Baylac, F-31059 Toulouse Cedex 9, France; Department of Radiology (F.d.P.), Santa Lucia Foundation, Rome; ITAB (F.d.P.), Department of Neuroscience Imaging and Clinical Science, G. D’Annunzio University, Chieti, Italy; and Leenaards Memory Center (J.-F.D.), Department of Clinical Neuroscience CHUV and University of Lausanne, Switzerland.

Go to Neurology.org for full disclosures. Funding information and disclosures deemed relevant by the authors, if any, are provided at the end of the article. The Article Processing Charge was paid by INSERM.

This is an open access article distributed under the terms of the Creative Commons Attribution-NonCommercial-NoDerivatives License 4.0 (CC BY-NC-ND), which permits downloading and sharing the work provided it is properly cited. The work cannot be changed in any way or used commercially.
} 
functional connectivity with the PMC was found to be correlated with the degree of clinical consciousness impairment across healthy controls and locked-in syndrome to minimally conscious state and vegetative state. ${ }^{9,10}$

The objective of the current study was to investigate the prognostic value of synchronicity indexes of the spontaneous activity in the PMC assessed in a cohort of brain-injured patients at the acute stage of coma. We hypothesized that in the challenging context of coma, the study of brain functional connectivity from PMC might decisively permit to (1) infer the involvement of large-scale functional networks coupled at rest with different PMC subregions (precuneus [PreCu] and posterior cingulate cortex $[\mathrm{PCC}]$ ) in consciousness generation, (2) identify the functional connectivity patterns that are specifically related to brain injury mechanisms, and (3) explore the extent to which PMC functional connectivity at rest could constitute an early and reliable predictor of neurologic recovery in this setting.

METHODS Standard protocol approvals, registrations, and patient consents. This study was approved by the ethics committee of the University Hospital of Toulouse, France ("Comite Consultatif pour la Protection des Personnes," CHU Toulouse, ID-RCB: 2012-A00009-34). Written informed consent was obtained directly from the healthy volunteers and from the legal surrogate of the patients. Clinical trials identifier: NCT01620957.

Participants. This multicenter study was undertaken in 4 intensive critical care units affiliated with the University Teaching Hospital (Toulouse, France) between January 2013 and February 2014. We compared 31 patients with severe brain injury who met the clinical definition of coma (Glasgow Coma Scale score at the admission to hospital $<8$ with motor responses $<6 ; 16$ patients with traumatic and 15 with anoxic brain injury; age range: $23-51$ years) with 10 age-matched healthy volunteers (range: 24-45 years). All patients were prospectively recruited and managed according to standard of care recommendations. ${ }^{11}$ To avoid confounding factors, all patient assessments were conducted at least 2 days ( $4 \pm 2$ days) after complete withdrawal of sedative drug therapy and performed under normothermic conditions. In patients, standardized clinical examination was performed by raters blinded to neuroimaging data (Glasgow Coma Scale ${ }^{12}$ and the Full Outline of Unresponsiveness ${ }^{13}$ on the day of scanning and 3 months later using the Coma Recovery ScaleRevised $\left.{ }^{14}\right)$. Table 1 reports demographic and clinical characteristics of the patients.

Imaging procedures. Acquisitions. In all participants, $11 \mathrm{mi}-$ nutes of resting-state fMRI was acquired on a $3 \mathrm{~T}$ magnetic resonance scanner (Intera Achieva; Philips, Best, the Netherlands). Two hundred fifty multislice T2*-weighted fMRIs were obtained with a gradient echo-planar sequence using axial slice orientation (37 slices; voxel size: $2 \times 2 \times 3.5 \mathrm{~mm}^{3}$; repetition time $=2,600$ milliseconds; echo time $=30$ milliseconds; flip angle $=90^{\circ}$; field of view $=240 \mathrm{~mm}$ ). A 3-dimensional T1-weighted sequence (inplane resolution $1 \times 1 \times 1 \mathrm{~mm}, 160$ contiguous slices) was also acquired in the same session. Monitoring of vital measures was performed by a senior intensivist (S.S., C.V.) throughout the experiment.

Seed-region integrity. Two PMC subregions were defined as regions of interest (ROIs): PCC and PreCu (figure e-1 on the Neurology ${ }^{\circledR}$ Web site at Neurology.org). They covered the entire area of the corresponding anatomical region defined by the Automated Anatomical Labeling atlas. ${ }^{15}$ The structural integrity of these seeds was guaranteed by visual assessment on T1 imaging. Four patients ( 3 with traumatic and 1 with anoxic brain injury) showed significant anatomical anomalies in these regions and were excluded from further analysis. In the remaining participants of the cohort, the $\mathrm{T}^{*}$ mean values of voxels located in the seed region were extracted from the T2* mean images and 2-sample $t$ tests were performed to compare values measured in the control and patient groups; no significant difference was observed between the groups (table e-1).

Preprocessing. Data analysis was performed using SPM8 (http://www.fil.ion.ucl.ac.uk/spm/) and the CONN-fMRI functional connectivity toolbox, version 13.p (http://www.nitrc. org/projects/conn). ${ }^{16}$ Echo-planar images were realigned, coregistered, and normalized to the Montreal Neurological Institute echo-planar imaging template, and smoothed using an 8.0-mm full-width at half maximum isotropic gaussian filter. The head movements estimated during realignment were included in the model as confounding factors. In addition, using the aCompCor method, ${ }^{17}$ covariates were included in a principal components analysis (PCA) reduction (3 dimensions) of the signal from white matter and CSF voxels. The residual blood oxygen level-dependent (BOLD) time series was bandpass-filtered over a low-frequency window of interest $(0.009 \mathrm{~Hz}<\mathrm{f}<0.08 \mathrm{~Hz})$.

Hypothesis-driven-based analysis. In the first-level analysis, a correlation map was produced for each participant by extracting the residual BOLD time course from each ROI (PreCu and PCC, figure e-1). Pearson correlation coefficients were then calculated between both seed time course and the time course of all other voxels in the brain. Correlation coefficients were converted to normally distributed scores using Fisher transformation to allow for second-level general linear model analyses.

In the second-level analysis, we conducted a within-group analysis (1-sample $t$ test) to determine connectivity maps for each group from each ROI. These connectivity maps from each seed ROI from all participants were entered into a 2 -sample $t$ test, to identify regions with different PCC connectivity or PreCu connectivity across the whole group of patients and healthy controls. We report regions that survived a height threshold of false discovery rate (FDR)-corrected $p<0.001$ at the voxel level and an extent threshold of family-wise error-corrected $p<0.05$ at the cluster level. We further explored our data until a threshold at FDR-corrected $p<$ 0.05 at the voxel level. The connectivity value (Fisher $z$ score) between the seed (PCC and/or PreCu) and each ROI was extracted from the seed connectivity map from each participant, to further segregate between positively vs negatively correlated ROIs relative to each seed region. Fisher $z$ values extracted from the ROIs were converted back to correlation coefficients ( $r$ values) for reporting purposes.

To specifically address the influence of the etiology of brain injury, 3 additional 2-sample $t$ tests were conducted on connectivity maps from each seed ROI: patients with traumatic brain injury (TBI) vs controls and anoxic patients vs controls and anoxic vs TBI. Only regions that survived a height threshold of 
Table 1 Population demographics and neurologic outcome

\begin{tabular}{|c|c|c|c|c|c|c|c|c|}
\hline Patient & Sex & Age, y & Etiology & Time to MRI, d & $\begin{array}{l}\text { GCS score } \\
\text { at scan }\end{array}$ & $\begin{array}{l}\text { FOUR score } \\
\text { at scan }\end{array}$ & $\begin{array}{l}\text { CRS-R score } \\
\text { at } 3 \mathrm{mo}\end{array}$ & Structural MRI findings \\
\hline 1 & M & 35 & CA & 7 & 7 & 6 & 6 (VS) & High signal of the thalami bilaterally \\
\hline 2 & M & 64 & $\mathrm{TBI}$ & 4 & 5 & 5 & 14 (MCS) & Cortical microhemorrhages (frontal lobes) \\
\hline 3 & M & 70 & $\mathrm{CA}$ & 5 & 4 & 2 & 4 (VS) & $\begin{array}{l}\text { Diffuse hypoxic-ischemic injury (thalami, } \\
\text { occipital lobes, parietal lobes) }\end{array}$ \\
\hline 4 & $\mathrm{~F}$ & 58 & TBI & 3 & 3 & 1 & 9 (VS) & Diffuse axonal injury \\
\hline 6 & M & 34 & $\mathrm{CA}$ & 5 & 4 & 2 & 20 (REC) & $\begin{array}{l}\text { Mild increase in signal in thalami and } \\
\text { hippocampi }\end{array}$ \\
\hline 7 & $\mathrm{~F}$ & 70 & TBI & 5 & 5 & 4 & 8 (VS) & $\begin{array}{l}\text { Cortical contusions (temporal lobes) and } \\
\text { subcortical gray matter lesion (thalamus) }\end{array}$ \\
\hline 8 & $\mathrm{~F}$ & 69 & $\mathrm{TBI}$ & 3 & 5 & 3 & 7 (VS) & Diffuse axonal injury \\
\hline 12 & $\mathrm{~F}$ & 48 & $\mathrm{CA}$ & 5 & 3 & 1 & 15 (MCS) & $\begin{array}{l}\text { Diffuse ischemic changes (thalami, occipital } \\
\text { lobes) }\end{array}$ \\
\hline 13 & M & 59 & TBI & 2 & 4 & 4 & 16 (MCS) & Cortical, brainstem and cerebellar lesions \\
\hline 14 & $\mathrm{~F}$ & 47 & $\mathrm{CA}$ & 5 & 5 & 6 & 8 (VS) & No abnormality \\
\hline 15 & M & 56 & TBI & 3 & 5 & 5 & 6 (VS) & Diffuse axonal injury and brainstem lesion \\
\hline 16 & M & 56 & TBI & 4 & 6 & 6 & 21 (REC) & Cortical contusions \\
\hline 17 & $\mathrm{~F}$ & 35 & $\mathrm{CA}$ & 6 & 5 & 4 & 22 (REC) & High signal in thalami bilaterally \\
\hline 18 & M & 58 & $\mathrm{CA}$ & 6 & 6 & 5 & 7 (VS) & No abnormality \\
\hline 25 & M & 21 & TBI & 6 & 6 & 5 & 14 (MCS) & $\begin{array}{l}\text { Cortical contusions (temporal lobes) and } \\
\text { diffuse axonal injury }\end{array}$ \\
\hline 26 & $\mathrm{~F}$ & 58 & TBI & 9 & 6 & 7 & 23 (REC) & Cortical contusions (frontal lobes) \\
\hline 27 & M & 49 & $\mathrm{CA}$ & 8 & 5 & 4 & 13 (MCS) & $\begin{array}{l}\text { High increase in signal in thalami and } \\
\text { hippocampi }\end{array}$ \\
\hline
\end{tabular}

Abbreviations: $\mathrm{CA}=$ cardiac arrest; CRS-R = Coma Recovery Scale-Revised; FOUR = Full Outline of Unresponsiveness; GCS = Glasgow Coma Scale; $\mathrm{MCS}=$ minimally conscious state; $\mathrm{TBI}=$ traumatic brain injury; VS = vegetative state.

FDR-corrected $p<0.001$ at the voxel level and an extent threshold of family-wise error-corrected $p<0.05$ at the cluster level were reported. Finally, a null conjunction was performed between TBI vs controls and anoxic vs controls. We used FDR correction with $p<0.05$ at a voxel level.

Behavioral data are expressed as median and range. Data distributions were compared with Kruskal-Wallis test, and the statistical dependence between 2 variables was assessed using Spearman rank correlation coefficient.

Data-driven analysis. To provide an additional methodologic control, we included a complementary data-driven spatial independent component analysis ${ }^{18}$ to decompose data into statistically independent spatial and temporal components ${ }^{19}$ (http://icatb.sourceforge.net). A standard template of the defaultmode network (DMN) was used to identify network components on a subject-by-subject basis. ${ }^{20}$ SPM8 (http://www.fil.ion.ucl.ac. $\mathrm{uk} / \mathrm{spm} /$ ) was used for the statistical comparison of images. These ancillary data have been used to create figure e- 2 .

RESULTS Plasticity of brain connections after injury. In figure 1, we report group-level results of seed-based connectivity analyses obtained when PreCu and PCC were considered in turn as seed. The obtained comatose patient's topography resembled the one reported in healthy controls, involving correlated brain areas (figure 1, red) previously described as being part of internally directed cognition networks ${ }^{21}$ : medial prefrontal cortex (mPFC), temporoparietal junction, and parahippocampal and superior frontal gyri. In 


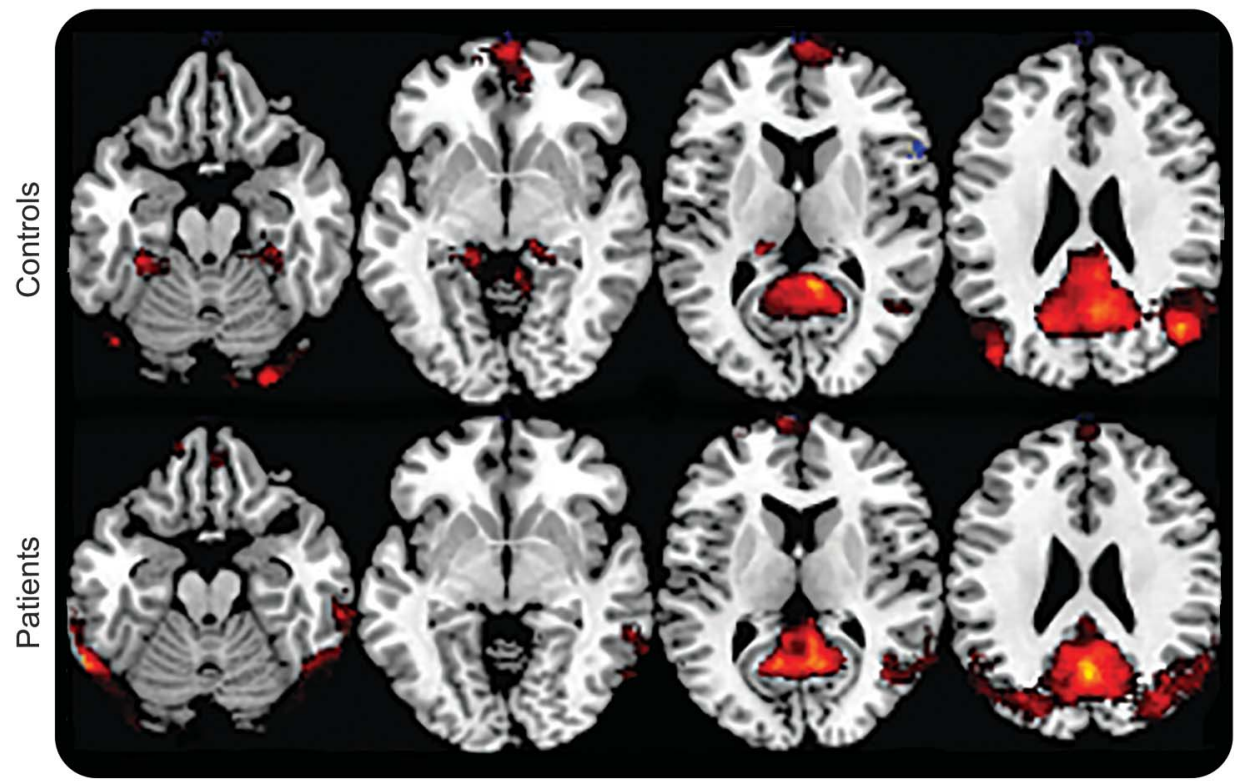

PreCu spontaneously synchronized network

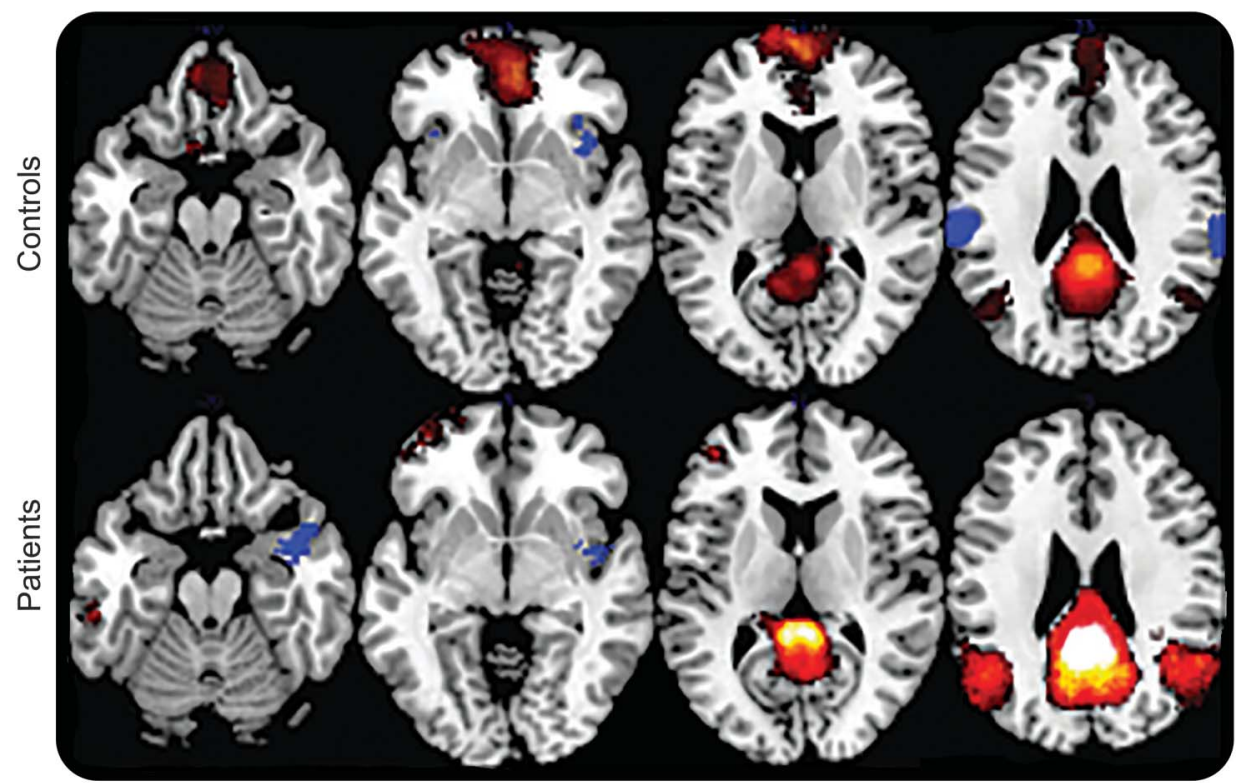

PCC spontaneously synchronized network

Whole-brain functional connectivity maps of covariance were computed in both groups (i.e., comatose patients and controls) following a hypothesis-driven approach. Two seed-based structures were analyzed: posterior cingulate cortex (PCC) and precuneus (PreCu). Color intensity depicts level of synchronicity (red and blue for positive and negative temporal signals correlations, respectively). All $p$ values are corrected for false discovery rate at the whole brain level ( $p$ value $<0.0001$, corrected for false discovery rate).

addition, we also observed in both patient and control groups a set of brain areas that were spontaneously anticorrelated with PCC and PreCu (figure 1, blue). Such anticorrelated network bilaterally involved the supramarginal gyrus (SMG) and the insulae, regions that are known to take part in networks supporting externally directed cognition. ${ }^{22}$

In addition, to quantify the observational similarity between healthy control and patient connectivity maps, we computed the statistical differences between the obtained networks (figure 2, in purple and green significant positive and negative differences, respectively). When considering PCC as seed region, we observed a significant and specific functional connectivity change in patients who were comatose relative to healthy controls (figure 2A). It is worth noting that these functional connectivity disruptions from PCC involved spontaneously correlated (e.g., mPFC) and 
Figure 2 Comparison between intrinsic PCC synchronized networks identified in patients who were comatose and controls
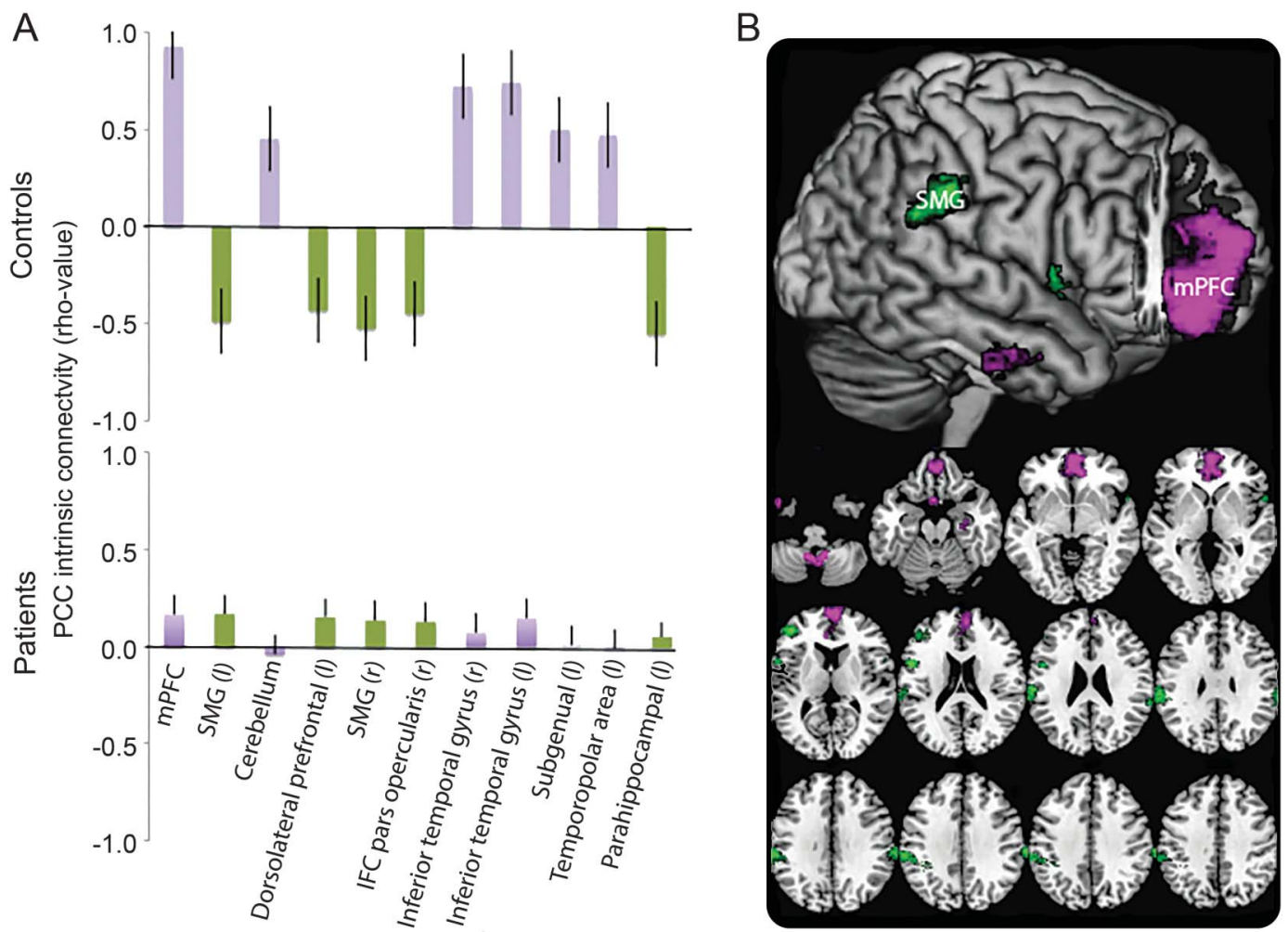

(A) Color intensity depicts differences of synchronicity between both groups (purple and green for positive and negative differences, respectively). (B) Spatial distribution of differences of temporal synchronicity values between patients and controls. All $p$ values are corrected for false discovery rate at the whole brain level ( $p$ value $<0.0001$, corrected for false discovery rate). IFC = inferior frontal cortex; $\mathrm{mPFC}=$ mesial prefrontal cortex (BA9); $\mathrm{PCC}=$ posterior cingulate cortex; SMG = supramarginal gyrus (BA40).

anticorrelated (e.g., SMG) brain areas (figure 2B). A data-driven spatial independent component analysis, used as a methodologic control, confirms that DMN was indeed much less integrated in patients who were comatose compared with controls (figure e-2).

In contrast, no such group differences were observed for connectivity of the $\mathrm{PreCu}$ seed region $(p<0.05$, FDR-corrected), suggesting the preservation of a global cortical network orchestrated by Pre$\mathrm{Cu}$ over time in resting state during coma.

Effect of brain injury mechanism. It is worth noting that no difference was found between the resting-state networks obtained from patients of traumatic vs anoxic origin at the defined statistical threshold $(p<$ 0.05 , FDR-corrected). Using a lower threshold ( $p<$ 0.001 , uncorrected), only 2 clusters of voxel were identified: the left somatosensory associative cortex (BA7) and the retrosplenial cingulate cortex (BA29). Of note, largely overlapping networks were observed when comparing intrinsic connectivity networks from coma patients of traumatic vs anoxic origin (figure 3 ). A conjunction analysis confirmed and extended this finding (figure e-3), suggesting a common pattern of neural communication disturbance in coma patients, notwithstanding etiology. To summarize, we observed at the same level, in both traumatic and anoxic patients, a significant disturbance in coactivation of distant regions from PCC, in particular with the midline forebrain areas.

Prognostic value. In this behaviorally homogeneous cohort of patients who were comatose (table 1), we observed that the functional connectivity strength between PCC-mPFC assessed at the acute stage where patients were scanned, was significantly different between comatose patients who went on to recover and those who eventually scored an unfavorable outcome 3 months after the brain injury (figure 4A). In addition, the linear regression between patients' outcome score on CRS-R and the PCC-mPFC activity coupling at rest substantiated a significant link between PCC-mPFC functional connectivity that was recorded promptly after brain injury and patients' neurologic recovery evaluated later (figure 4B).

DISCUSSION Theoretical conceptualizations of the neural bases of conscious processes suggest that both local and long-range recursive processing loops through cortical hubs permit global availability of 
Figure $3 \quad$ Intrinsic PCC synchronized networks identified in comatose patients with anoxic and traumatic injury compared with controls

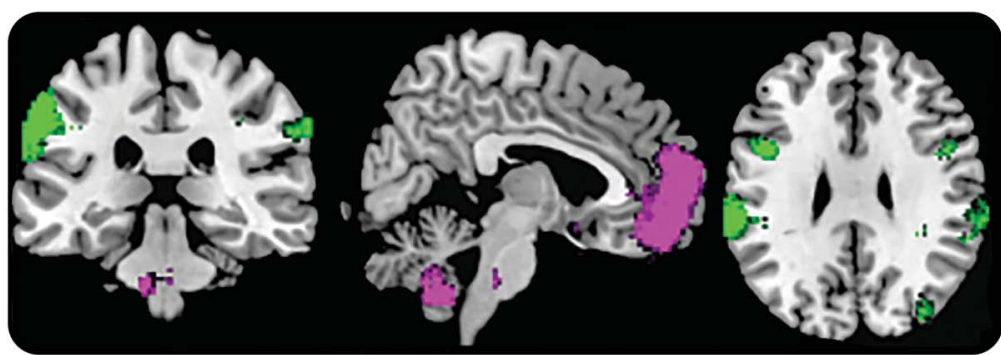

Traumatic vs control

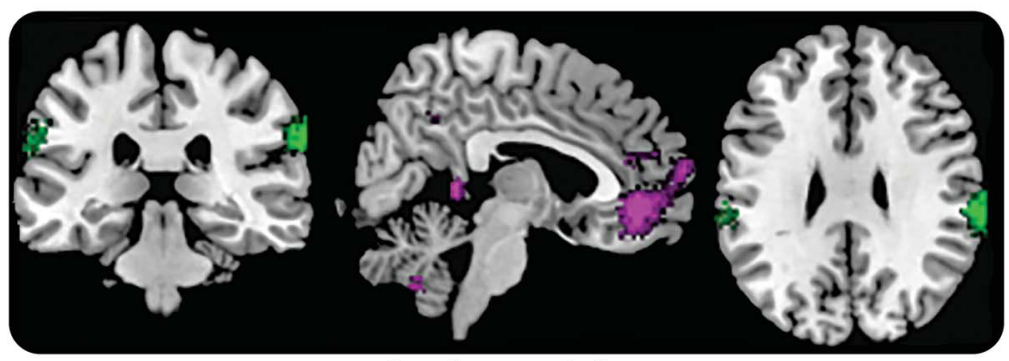

Anoxic vs control

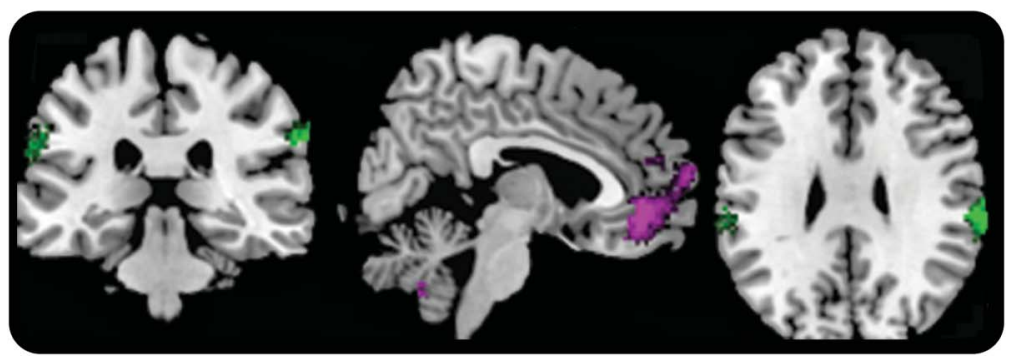

Traumatic vs control $\cap$ Anoxic vs control

One seed-based structure was analyzed (PCC) ( $p$ value $<0.0001$, corrected for false discovery rate). Both etiologic subgroups are represented (i.e., traumatic and anoxic mechanism, upper and middle panel, respectively). In addition, overlapping resting-state networks, in which activity is spontaneously synchronized with PCC in both traumatic and anoxic comatose patients compared with controls, are depicted in the lower panel. PCC = posterior cingulate cortex. coma, has a significant predictive value of further neurologic recovery. Our results support previous reports suggesting that cortico-cortical spontaneous synchronization may be used as an efficient diagnostic classifier for vegetative state and minimally conscious state $^{8,9}$ and pave the way for future longitudinal studies aiming to fully describe the structural and functional network dysfunction induced by severe brain injury from acute comatose states throughout the recovery phase.

It must be highlighted that most of the studies that have analyzed the functional contribution of the PMC in patients with acquired disorders of consciousness explored this portion of the parietal lobe as an indivisible structure (PCC/PreCu). ${ }^{8,9,18,23} \mathrm{Nev-}$ ertheless, a growing body of research has suggested an important structural and functional heterogeneity within this large region. Cytoarchitectonically, the PCC is characterized as paralimbic cortex, exhibiting a transitional cell architecture organized in 2 subregions, one dorsal (areas d23a, d23b, 23d, anterior 31) and one ventral (v23a and v23b, posterior 31). ${ }^{24}$ In addition, tract-tracing studies conducted in nonhuman primates ${ }^{25}$ and diffusion-tensor tractography in humans ${ }^{26}$ have clearly identified structural connections between the ventral PCC and the medial temporal lobes, as well as connections from more dorsal PCC to the mPFC along the cingulum bundle. ${ }^{27}$ Finally, PCC complex cognitive function has been recently synthesized into an "Arousal, Balance of Breath of Attention" model. ${ }^{2}$ However, PreCu (BA7) is characterized by a fully differentiated isocortex, ${ }^{28}$ has reciprocal cortico-cortical connections with the adjacent areas of the PMC, namely, the frontal lobes, the supplementary motor area, ${ }^{29}$ together with its own major subcortical connections with the basal ganglia and the brainstem. ${ }^{30}$ Functional neuroimaging studies appear to converge with evidence for a functional role of PreCu involving self-centered imagery strategies and subserving successful episodic memory retrieval. ${ }^{31}$ Our data are in line with this PMC heterogeneity, since we found significant differences of connectivity changes between intrinsic networks depending on whether the considered seed region was PCC or PreCu.

Regarding the temporally coupled networks seeded in PCC at rest, we identified a 2-fold connectivity disruption pattern. First, we observed a midline decoupling of PCC and mPFC suggesting network sensitivity to changes in level of consciousness. Of note, our results agree with previous reports suggesting a link between internal awareness and the activity of mPFC and PCC, ${ }^{22}$ as a stepwise reduction of connectivity between these midline structures in different sleep states ${ }^{6}$ and loss of the normal top-down corticocortical communication between them observed with 


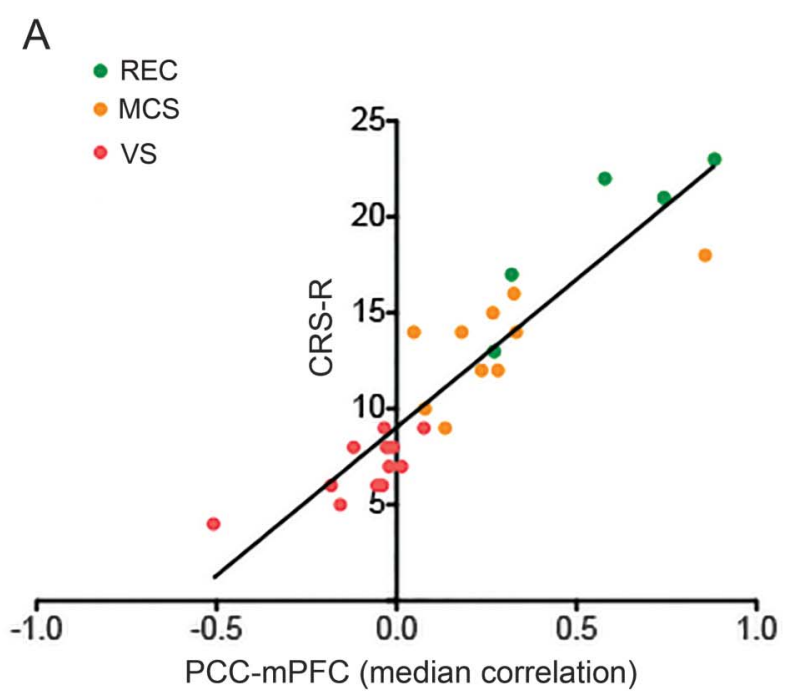

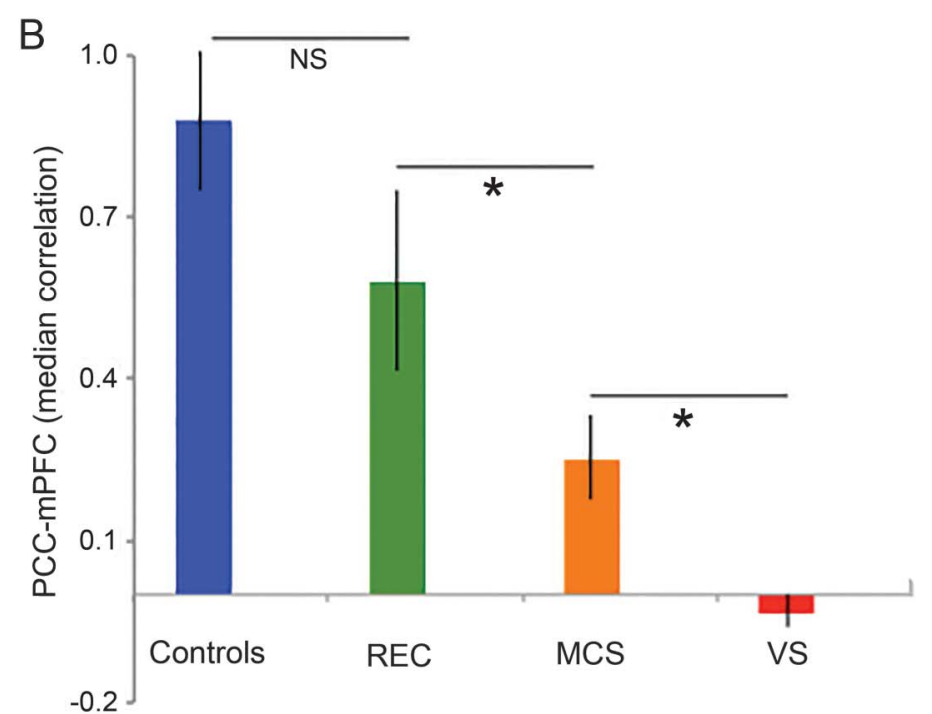

The linear regression between patient outcome CRS-R and early-recorded PCC-mPFC coupling at rest (A) suggested a significant link between PCC-mPFC functional connectivity and patient neurologic recovery (Spearman $\rho=0.93, p<0.003$ ). Functional connectivity strength between PCC-mPFC assessed at scan time (B) was significantly different between comatose patients who recovered consciousness (REC) and those who evolved toward a minimally conscious state (MCS) or a vegetative state (VS) 3 months after the brain injury (Kruskal-Wallis test, $p<0.001$ ). PCC-mPFC synchronicity at rest was not different between REC and controls. $\mathrm{mPFC}=$ medial prefrontal cortex; NS = not significant; $\mathrm{PCC}=$ posterior cingulate cortex.

propofol sedation. ${ }^{32}$ This finding is also convergent with the hypothesis of a dysfunctional mesocircuit, ${ }^{33}$ which posits that the anterior forebrain function is markedly downregulated in all severe brain injures as a result of long-range cerebral disconnections. Second, we detected in patients a deficit of the normal correlation over time between PCC and lateral parietal cortices (mainly SMG) that have been previously associated with external awareness. ${ }^{34}$ We suggest that our data could depict breakdown of the dynamic reciprocal interaction between internal and external awareness systems during coma and highlight the pivotal role of PCC for "tuning" the whole-brain metastable status. ${ }^{35}$

Remarkably, the alteration of coordination over time between PCC and midline forebrain areas was found to be at a similar level in both patients with traumatic and patients with anoxic injury. Converging structural neuroimaging data have shown specific perturbations of white matter pathways between PCC and $\mathrm{mPFC}$ in both traumatic brain injury ${ }^{36}$ and anoxia. ${ }^{37}$ However, we submit the alternative and complementary account according to which the anterior-posterior disconnection pattern jointly observed in both anoxic and traumatic groups reflects remote and diffuse functional imbalance throughout the whole neuronal system (i.e., diaschisis). Future studies combining structural and functional data will be needed to specifically address this issue.

The measurement of PreCu spontaneous activity synchronicity at rest, compared between patients who were comatose and controls allowed us to identify a robust and autonomous intrinsic network, encompassing the areas implicated in the DMN. We observed that even in this extreme pathologic condition of consciousness disruption, the functional architecture of this PreCu-based network as a whole was maintained. This important point could be contextualized with the experimental background suggesting that the DMN may be needed for consciousness to occur but is not sufficient on its own to elicit awareness. ${ }^{38}$ It seems that this functional connectivity pattern linked to PreCu spontaneous activity transcends the level of consciousness, and could probably be considered as a physiologic baseline.

Several limitations of our study should be recognized. First, the number of patients is relatively small and our results need to be prospectively validated in larger cohorts. Second, there is a possibility that our fMRI findings could change depending on the time of scanning in relation to the time of injury. ${ }^{18}$ This important point highlights the interest of early and repeated neuroimaging assessments in this setting, intended to accurately identify and monitor the brain network functional changes that are specifically related to consciousness emergence from coma.

To summarize, the reorganization of PCC-centered, spontaneously synchronized, large-scale networks seems to be implicated in the loss of external and internal self-centered awareness observed during coma, largely independent of its etiology. The level of functional connectivity between PCC and mPFC appears to be related 
to patient neurologic outcome. Future work should further explore brain intrinsic network dysfunctions in larger patient cohorts, aiming to improve patient diagnosis and early prognostication and enable the development of innovative network-based personalized treatments.

\section{AUTHOR CONTRIBUTIONS}

S.S. takes responsibility for the content of the manuscript, including the data and analysis. He conceived the study and has personally made contributions to the design of the study, the acquisition of data, and the analysis and interpretation of data. C.V., B.R., T.G., T.S., V.B., and O.F. have substantially participated in the data acquisition. P.P. and I.L. have made contribution to the design of the study. P.P., F.d.P., and J.-F.D have contributed to the conception and analysis of data. S.S., F.d.P., J.-F.D., and P.P. wrote the manuscript. All authors have revised the submitted manuscript critically for important intellectual content and they have provided final approval of this version to be published. All authors agree to the conditions outlined in the Guide for Authors.

\section{ACKNOWLEDGMENT}

The authors thank the technicians and engineers of the Neurocampus and Brain Imaging Center of Purpan (Helene Gros, Nathalie Vayssiere) and the medical staff of the Critical Care Units of the University Teaching Hospital of Toulouse (Mylene Terrade, Fanny Vardon, Lionel Kheruel, Dalinda Ait Aissa) for their active participation in the MRI studies in comatose patients.

\section{STUDY FUNDING}

Funding was provided by the Association des Traumatisés du Crâne et de la Face, Institut des Sciences du Cerveau de Toulouse, and Institut National de la Santé et de la Recherche Médicale. The funding sources had no role in the study design, data collection, data analysis, data interpretation, or writing of this report.

\section{DISCLOSURE}

The authors report no disclosures relevant to the manuscript. Go to Neurology.org for full disclosures.

Received April 8, 2015. Accepted in final form August 10, 2015.

\section{REFERENCES}

1. Cavanna AE, Trimble MR. The precuneus: a review of its functional anatomy and behavioural correlates. Brain 2006;129:564-583.

2. Leech R, Sharp DJ. The role of the posterior cingulate cortex in cognition and disease. Brain 2014;137:12-32.

3. Andrews-Hanna JR, Reidler JS, Sepulcre J, Poulin R, Buckner RL. Functional-anatomic fractionation of the brain's default network. Neuron 2010;65:550-562.

4. Dehaene S, Changeux JP. Experimental and theoretical approaches to conscious processing. Neuron 2011;70:200-227.

5. Gusnard DA, Akbudak E, Shulman GL, Raichle ME. Medial prefrontal cortex and self-referential mental activity: relation to a default mode of brain function. Proc Natl Acad Sci USA 2001;98:4259-4264.

6. Horovitz SG, Braun AR, Carr WS, et al. Decoupling of the brain's default mode network during deep sleep. Proc Natl Acad Sci USA 2009;106:11376-11381.

7. Amico E, Gomez F, Di Perri C, et al. Posterior cingulate cortex-related co-activation patterns: a resting state $\mathrm{fMRI}$ study in propofol-induced loss of consciousness. PLoS One 2014;9:e100012.

8. Silva S, Alacoque X, Fourcade O, et al. Wakefulness and loss of awareness: brain and brainstem interaction in the vegetative state. Neurology 2010;74:313-320.
9. Vanhaudenhuyse A, Noirhomme Q, Tshibanda LJ, et al. Default network connectivity reflects the level of consciousness in non-communicative brain-damaged patients. Brain 2010;133:161-171.

10. Soddu A, Vanhaudenhuyse A, Demertzi A, et al. Resting state activity in patients with disorders of consciousness. Funct Neurol 2011;26:37-43.

11. Menon DK. Cerebral protection in severe brain injury: physiological determinants of outcome and their optimisation. Br Med Bull 1999;55:226-258.

12. Plum F, Posner J. The Diagnosis of Stupor and Coma. Philadelphia: F.A. Davis; 1982.

13. Wijdicks EF, Bamlet WR, Maramattom BV, Manno EM, McClelland RL. Validation of a new coma scale: the FOUR score. Ann Neurol 2005;58:585-593.

14. Boly M, Massimini M, Tononi G. Theoretical approaches to the diagnosis of altered states of consciousness. Prog Brain Res 2009;177:383-398.

15. Tzourio-Mazoyer N, Landeau B, Papathanassiou D, et al. Automated anatomical labeling of activations in SPM using a macroscopic anatomical parcellation of the MNI MRI single-subject brain. Neuroimage 2002;15:273-289.

16. Whitfield-Gabrieli S, Nieto-Castanon A. Conn: a functional connectivity toolbox for correlated and anticorrelated brain networks. Brain Connect 2012;2:125-141.

17. Behzadi Y, Restom K, Liau J, Liu TT. A component based noise correction method (CompCor) for BOLD and perfusion based fMRI. Neuroimage 2007;37:90-101.

18. Norton L, Hutchison RM, Young GB, Lee DH, Sharpe MD, Mirsattari SM. Disruptions of functional connectivity in the default mode network of comatose patients. Neurology 2012;78:175-181.

19. Calhoun VD, Adali T, Pearlson GD, Pekar JJ. A method for making group inferences from functional MRI data using independent component analysis. Hum Brain Mapp 2001;14:140-151.

20. Garrity AG, Pearlson GD, McKiernan K, Lloyd D, Kiehl KA, Calhoun VD. Aberrant "default mode" functional connectivity in schizophrenia. Am J Psychiatry 2007;164:450-457.

21. Raichle ME, MacLeod AM, Snyder AZ, Powers WJ, Gusnard DA, Shulman GL. A default mode of brain function. Proc Natl Acad Sci USA 2001;98:676-682.

22. Dixon ML, Fox KC, Christoff K. A framework for understanding the relationship between externally and internally directed cognition. Neuropsychologia 2014; 62:321-330.

23. Laureys S, Goldman S, Phillips C, et al. Impaired effective cortical connectivity in vegetative state: preliminary investigation using PET. Neuroimage 1999;9:377-382.

24. Vogt BA, Laureys S. Posterior cingulate, precuneal and retrosplenial cortices: cytology and components of the neural network correlates of consciousness. Prog Brain Res 2005;150:205-217.

25. Vogt BA, Vogt L, Farber NB, Bush G. Architecture and neurocytology of monkey cingulate gyrus. J Comp Neurol 2005;485:218-239.

26. Hagmann P, Cammoun L, Gigandet X, et al. Mapping the structural core of human cerebral cortex. PLoS Biol 2008; 6:e159.

27. Greicius MD, Supekar K, Menon V, Dougherty RF. Resting-state functional connectivity reflects structural connectivity in the default mode network. Cereb Cortex 2009;19: 72-78. 
28. Pandya DN, Seltzer B. Intrinsic connections and architectonics of posterior parietal cortex in the rhesus monkey. J Comp Neurol 1982;204:196-210.

29. Cavada C, Goldman-Rakic PS. Posterior parietal cortex in rhesus monkey: I: parcellation of areas based on distinctive limbic and sensory corticocortical connections. J Comp Neurol 1989;287:393-421.

30. Petrides M, Pandya DN. Projections to the frontal cortex from the posterior parietal region in the rhesus monkey. J Comp Neurol 1984;228:105-116.

31. Fletcher PC, Frith CD, Baker SC, Shallice T, Frackowiak RS, Dolan RJ. The mind's eye: precuneus activation in memoryrelated imagery. Neuroimage 1995;2:195-200.

32. Boly M, Moran R, Murphy M, et al. Connectivity changes underlying spectral EEG changes during propofol-induced loss of consciousness. J Neurosci 2012;32:7082-7090.

33. Schiff ND. Recovery of consciousness after brain injury: a mesocircuit hypothesis. Trends Neurosci 2010;33:1-9.
34. Vanhaudenhuyse A, Demertzi A, Schabus M, et al. Two distinct neuronal networks mediate the awareness of environment and of self. J Cogn Neurosci 2011;23:570-578.

35. Hellyer PJ, Shanahan M, Scott G, Wise RJ, Sharp DJ, Leech R. The control of global brain dynamics: opposing actions of frontoparietal control and default mode networks on attention. J Neurosci 2014;34:451-461.

36. Palacios EM, Sala-Llonch R, Junque C, et al. White matter/gray matter contrast changes in chronic and diffuse traumatic brain injury. J Neurotrauma 2013;30: 1991-1994.

37. Luyt CE, Galanaud D, Perlbarg V, et al. Diffusion tensor imaging to predict long-term outcome after cardiac arrest: a bicentric pilot study. Anesthesiology 2012;117: 1311-1321.

38. Vincent JL, Patel GH, Fox MD, et al. Intrinsic functional architecture in the anaesthetized monkey brain. Nature 2007;447:83-86.

\section{Share Your Artistic Expressions in Neurology 'Visions'}

AAN members are urged to submit medically or scientifically related artistic images, such as photographs, photomicrographs, and paintings, to the "Visions" section of Neurology ${ }^{\circledR}$. These images are creative in nature, rather than the medically instructive images published in the NeuroImages section. The image or series of up to six images may be black and white or color and must fit into one published journal page. Accompanying description should be 100 words or less; the title should be a maximum of 96 characters including spaces and punctuation.

Learn more at www.aan.com/view/Visions, or upload a Visions submission at submit.neurology.org.

\section{Neurology ${ }^{\circledR}$ Genetics Call For Papers}

\section{Neurology \\ Gencinas}

Genetics

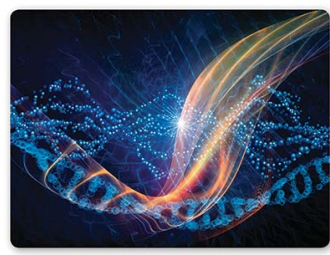

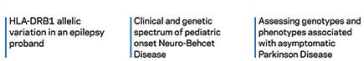

Neurology: Genetics is an open access, online only journal that provides neurologists with outstanding original contributions that elucidate the role of genetic and epigenetic variation in diseases and biological traits of the central and peripheral nervous system. We welcome all submissions. For more information on how to submit, visit http://www.neurology.org/site/ gen/gen2.xhtml. 


\section{Neurology}

\section{Disruption of posteromedial large-scale neural communication predicts recovery from coma}

Stein Silva, Francesco de Pasquale, Corine Vuillaume, et al.

Neurology 2015;85;2036-2044 Published Online before print November 11, 2015

DOI 10.1212/WNL.0000000000002196

This information is current as of November 11, 2015

\section{Updated Information \&} Services

\section{Supplementary Material}

\section{References}

Citations

Subspecialty Collections

Permissions \& Licensing

Reprints including high resolution figures, can be found at: http://n.neurology.org/content/85/23/2036.full

Supplementary material can be found at: http://n.neurology.org/content/suppl/2015/11/11/WNL.0000000000002 196.DC1

http://n.neurology.org/content/suppl/2016/07/18/WNL.0000000000002 196.DC2

This article cites 37 articles, 7 of which you can access for free at: http://n.neurology.org/content/85/23/2036.full\#ref-list-1

This article has been cited by 6 HighWire-hosted articles: http://n.neurology.org/content/85/23/2036.full\#\#otherarticles

This article, along with others on similar topics, appears in the following collection(s):

\section{Coma}

http://n.neurology.org/cgi/collection/coma

fMRI

http://n.neurology.org/cgi/collection/fmri

Prognosis

http://n.neurology.org/cgi/collection/prognosis

Information about reproducing this article in parts (figures,tables) or in its entirety can be found online at:

http://www.neurology.org/about/about_the_journal\#permissions

Information about ordering reprints can be found online:

http://n.neurology.org/subscribers/advertise

Neurology ${ }^{\circledR}$ is the official journal of the American Academy of Neurology. Published continuously since 1951, it is now a weekly with 48 issues per year. Copyright @ 2015 American Academy of Neurology. All rights reserved. Print ISSN: 0028-3878. Online ISSN: 1526-632X.

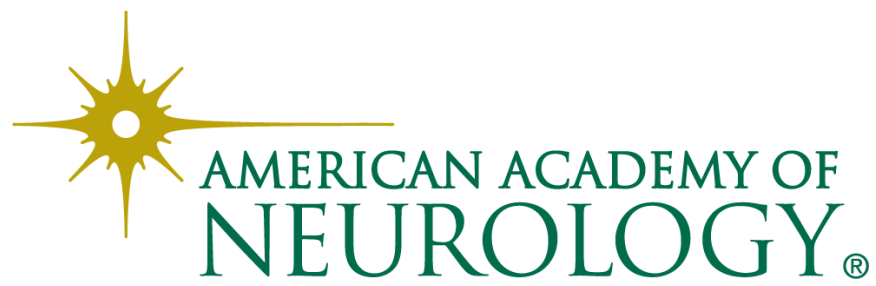

